\title{
Nuevo Enfoque en el Tratamiento de la Insuficiencia Placentaria Crónica
}

\author{
Antonio Soto-Yancés, M. D. - Profesor Asociado. \\ Victor Hernández A., M. D. - Instructor Asociado II \\ Rafael Guerrero B., M. D. - Profesor Titular \\ DEPARTAMENTO DE OBSTETRICIA Y GINECOLOGIA - UNIVERSIDAD DE CARTAGENA
}

\section{Introducción}

La Insuficiencia Placentaria Crónica, ha sido considerada como la causa fundamental del retardo del crecimiento fetal, y por ende, responsable del aumento de la morbi-mortalidad perinatal. La placenta produce, entre otras, una substancia que posee una actividad afín a la prolactina e inmunológicamente similar a la hormona del crecimiento humano, conocida como Lactógeno Placentario (HPL), cuya determinación sérica se considera en la actualidad como el mejor indicativo del funcionamiento placentario. Por otro lado, numerosos investigadores han demostrado la acción benéfica de los beta-adrenérgicos sobre la función placentaria y el aumento del flujo placentario. Con estos antecedentes realizamos el presente trabajo, utilizando la Orciprenalina como droga Betamimética.

\section{Lactógeno Placentario Humano Recuento Fisiológico}

EI Lactógeno Placentario Humano (HPL), es un polipéptido de cadena única con peso molecular de 21.000 y producido por el sincitio-trofoblasto, aunque es probable que se forme también en el citotrofoblasto, de vida media en sangre materna de 25 minutos.

La diversidad de procedimientos analíticos ha dado lugar a grandes diver- gencias en los valores de HPL en sangre, sin embargo todas las investigaciones demuestran que su concentración aumenta en forma continua durante el embarazo, hasta un máximo de 8 a 10 $\mathrm{mg} /$ litro a las 36 semanas y luego desciende paulatinamente hasta el término de la gestación. (Figura 1). En esta figura presentamos la curva de concentración sérica de HPL de acuerdo a los datos de Spellacy y cols., quien califica de Zona de Peligro Fetal (ZPF) los niveles inferiores a $4 \mathrm{mg} /$ litro a partir de las 30 semanas.

Figura No. 1

CURVA NORMAL DE HPL

$Y$ ZONA DE PELIGRO FETAL

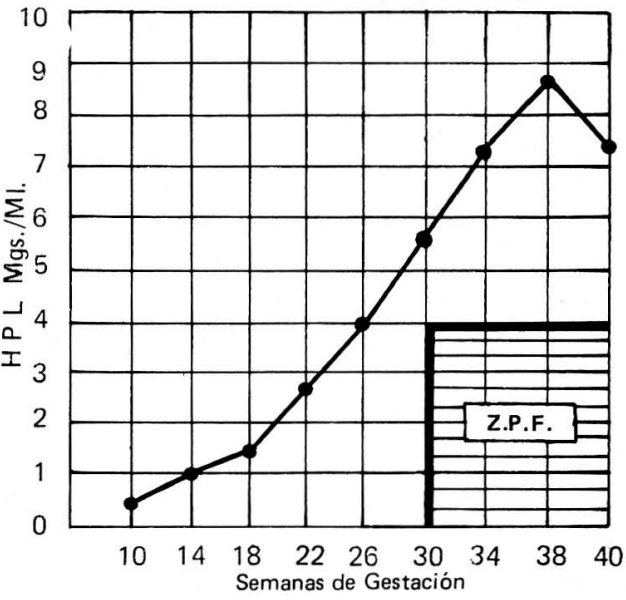




\section{Efectos Fisiológicos de los Betamiméticos sobre la Placenta}

Aun cuando el conocimiento de los betaadrenérgicos sobre la fisiología placentaria es todavía limitado, sus efectos han sido claramente apreciados por numerosos investigadores, quienes han demostrado una vasodilatación de los capilares vellositarios y aumento del flujo placentario, lo cual conlleva un aumento de los mecanismos de intercambio materno-fetal.

Por otro lado, se ha observado un incremento en los niveles de glucosa, debido a la difusión de una mayor cantidad de glucosa de la madre a la placenta y por ende al feto, lo cual teóricamente aumentaría los niveles de HPL como un mecanismo de "defensa". Sin embargo falta por comprobar las eventuales variaciones de otros parámetros metabólicos, como por ejemplo insulina, cortisol, etc., que podrían estar involucrados y que son en la actualidad motivo de investigación.

\section{Material y Métodos}

El estudio se realizó en el Departamento de Obstetricia y Ginecología de la Universidad de Cartagena y las pacientes fueron divididas en 3 grupos así:

GRUPO A. Pacientes normales, sin evidencia alguna de Patología Placentaria, que se tomaron como patrón para confeccionar la curva normal en nuestro medio.

GRUPO B. Pacientes con diagnóstico de Insuficiencia placentaria, que no recibieron drogas betamiméticas.

GRUPO C. Pacientes con diagnóstico de Insuficiencia Placentaria crónica tratadas con Orciprenalina, por vía oral $\mathrm{y} / \mathrm{o}$ sublingual, en dosis de 4 tabletas (80 mgs) diarios durante 2 semanas continuas. A todas las pacientes les fue practicada dosificación de HPL por lo menos en dos ocasiones, con intervalos de dos semanas. A las pacientes del grupo C se dosificó el HPL antes y después del tratamiento con Orciprenalina.

\section{Resultados}

GRUPO A. En este grupo constituido por 10 pacientes consideradas normales, encontramos niveles de HPL en progresivo aumento durante el embarazo. Los valores medios hallados durante las diferentes semanas de gestación son los siguientes:

30 - 32 Semanas - 5.2 microgramos/ $\mathrm{ml}$ - DS. 0.39

32 - 34 Semanas - 6.1 microgramos/ $\mathrm{ml}$ - DS. 0.57

34 - 36 Semanas - 8.4 microgramos/ $\mathrm{ml}$ - DS. 0.61

36 - 38 Semanas - 8.0 microgramos/ $\mathrm{ml}$ - DS. 0.53

La Figura 2 presenta la curva de concentración sérica de las pacientes normales en nuestro medio.

Figura No. 2

CONCENTRACION SERICA DE HPL EN PACIENTES NORMALES

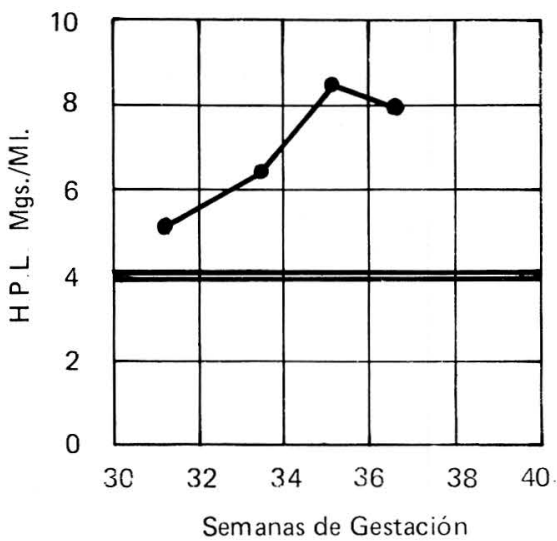

GRUPO B. Este grupo formado por 8 pacientes, con evidencia clínica de Insuficiencia Placentaria crónica, no recibieron tratamiento con Orciprenalina y en ellas se encontraron los siguientes valores medios:

1a. muestra (32 - 34 Semanas) - 4.2 microgramos $/ \mathrm{ml}$ - DS. 0.37 .

2a. muestra (34 - 36 Semanas) - 5.8 microgramos $/ \mathrm{ml}$ - DS. 0.51 
Figura No. 3

CONCENTRACION SERICA DE HPL EN

PACIENTES PATOLOGICAS NO TRATADAS

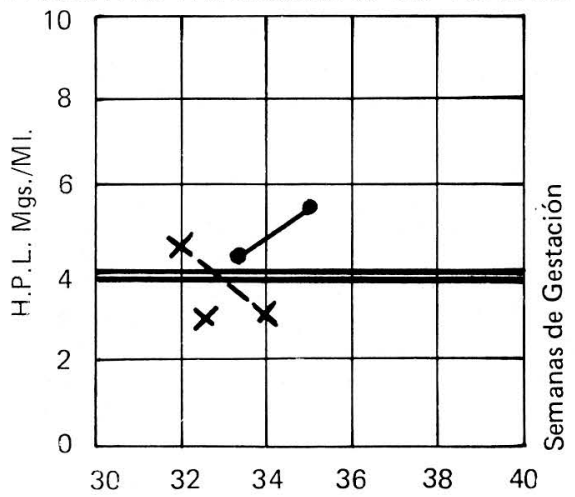

La Figura 3 ilustra la curva sérica de HPL en este Grupo.

Podemos observar que en este grupo el HPL, manifestó un incremento en los valores séricos, pero este nivel no logró sobrepasar la barrera de los $6 \mathrm{mi}$ crogramos $/ \mathrm{ml}$ a las 36 semanas. Por otro lado, en éste grupo se presentaron dos muertes fetales, en pacientes cuyos niveles estuvieron dentro de la llamada Zona de Peligro Fetal (ZPF).

GRUPO C. Constituido por 12 pacientes con evidencia de Insuficiencia Placentaria Crónica, las cuales fueron tratadas con Orciprenalina a las dosis y

Figura No. 4

CONCENTRACION SERICA DE HPL EN PACIENTES TRATADAS

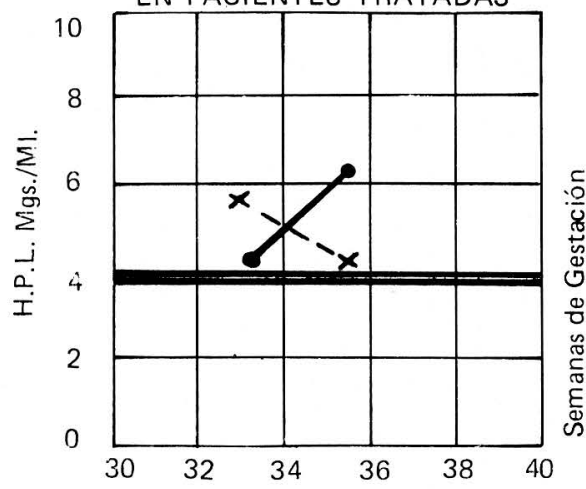

tiempo convenidos. Los valores medios de HPL encontrados en este grupo fueron los siguientes:

1a. muestra (pre-tratamiento) 32 - 34 semanas - Media: $4.2 \mathrm{microg} / \mathrm{ml}$ D.S. 0.35 .

2a. muestra (post-tratamiento) $34-36$ semanas - Media: $6.45 \mathrm{microg} / \mathrm{ml}$ D.S. 0.73 .

La Figura 4 presenta la curva sérica de HPL encontrada en este grupo.

Se puede observar que en este grupo se manifiesta un incremento significativo en los niveles de HPL, cuyos valores medios post-tratamiento rebasaran la línea de los 6 microgramos $/ \mathrm{ml}$ y en algunos casos la de los 7 microgramos $/ \mathrm{ml}$, pero sin llegar a los niveles considerados normales. En este grupo se presentó una muerte fetal, cuya causa atribuimos a una grave malformación congénita.

\section{Comentarios}

Los resultados obtenidos nos demuestran que:

Figura No. 5

CONCENTRACION SERICA DE H.P.L. CUADRO COMPARATIVO (MEDIA $\pm S$ )

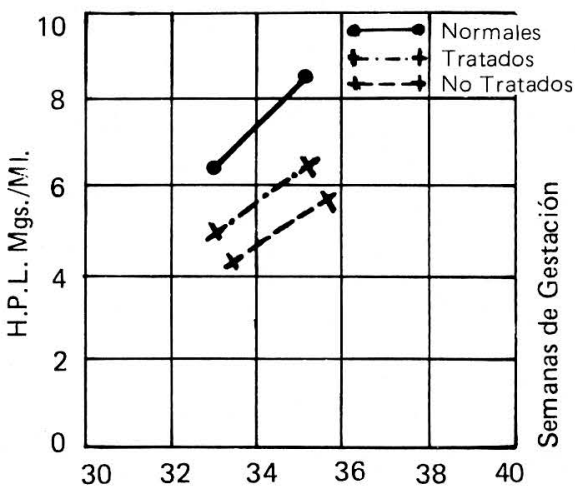

1. La dosificación de HPL, es un buen indicativo del funcionamiento placentario y que sus valores aumentan progresivamente durante las primeras 
36 semanas de la gestación y luego disminuyen progresivamente.

2. Que los valores de HPL por debajo de $5 \mathrm{microgramos} / \mathrm{ml}$ indican una deficiencia en la función placentaria y los inferiores a 4 microgramos por $\mathrm{ml}$, comprometen seriamente la vitalidad fetal.

3. Que el tratamiento con Orciprenalina aumenta significativamente los niveles séricos de HPL, sin llegar a los valores normales, pero coloca al feto fuera de la Zona de Peligro.

La Figura 5 presenta el cuadro comparativo de los 3 grupos, observando claramente el aumento de los niveles séricos de HPL en el grupo de pacientes tratadas con Orciprenalina.

\section{Resumen}

El estudio comprende tres grupos de pacientes divididos así:

GRUPO A: Pacientes Normales.

GRUPO B: Pacientes patológicas no tratadas con Orciprenalina.

GRUPO C: Pacientes patológicas tratadas con Orciprenalina.

En todos los grupos se dosificó el HPL, por lo menos en dos ocasiones y el Grupo C recibió Orciprenalina durante 2 semanas continuas.

Los resultados obtenidos permiten deducir que la administración de Orciprenalina produce un aumento significativo en los niveles de HPL, sérico y por consiguiente sería un nuevo recurso para tratar de disminuir la morbi-mortalidad fetal causada por la insuficiencia placentaria crónica.

\section{NEW APPROACH IN TREATING RECURRENT PLACENTAL INSUFFICIENCY}

\section{Summary}

The survey covers three groups of patients divided as follows:
Group $A=$ Normal Patients.

Group B $=$ Pathological Patients not treated with Orcyprenaline.

Group C $=$ Pathological Patients treated with Orcyprenaline.

HPL was dosed in all groups at least twice, and Group C received Orcyprenaline during two consecutive weeks.

From the results achieved it can be concluded that Orcyprenaline causes a significant increase in sericeous HPL levels; therefore, this can constitute a new way of trying to decrease fetal morbility and mortality produced by recurrent Placental Insufficiency.

\section{Bibliografía}

1 BILA, S.; SOLUVIC, V.; GENBACEV, O. Lactógeno Placentario sérico en pacientes con Retardo fetal intrauterino, IX Congreso Mundial de Obstetricia y Ginecología, Tokyo, 1979.

2 CABERO - ROURA, L.; Retardo del crecimiento intrauterino - Clínica e Investigación en Gineco Obstetricia, 5 - 122, 1978.

3 HOBBINS, J.; BERKOVITS, R., Lactógeno Placentario Humano; Estado actual. Clínicas Obst., y Ginec. Vol. 2 - págs. 387-97, 1978.

4 SPELLACY, W.; TEOH, E. y COLS., Valores de HPL en el manejo de los Embarazos de Alto Riesgo. Am. J. Obst. y Ginec. $109 \cdot 588,1971$

5 SOTO-YANCES, A., Normas terapéuticas en Obstetricia: Usos de la Orciprenalina, Tribuna-Médica, Dic. 1978.

6 SOTO-YANCES, A., DIAZ, M., DIAZ, T. Aceleración de la madurez pulmonar fetal con Orciprenalina. Rev. Col., Obst. y Ginec. XXIX, págs. 173-77, 1978. 